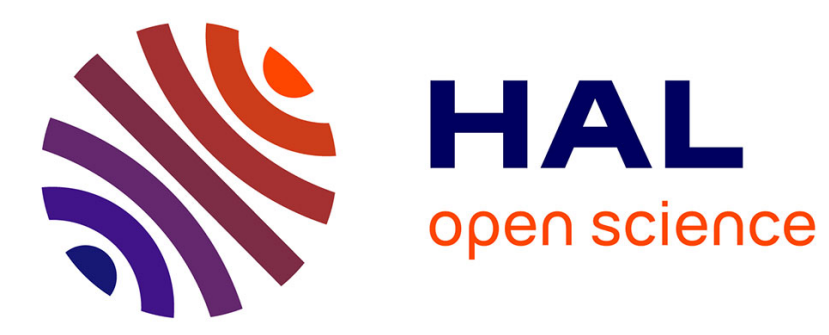

\title{
Les interrogatives indirectes en discours informel oral
}

Florence Lefeuvre, Nathalie Rossi-Gensane

\section{To cite this version:}

Florence Lefeuvre, Nathalie Rossi-Gensane. Les interrogatives indirectes en discours informel oral. Langue française, 2017, 196 (4), pp.51-74. 10.3917/lf.196.0051 . halshs-03360558

\section{HAL Id: halshs-03360558 \\ https://shs.hal.science/halshs-03360558}

Submitted on 30 Sep 2021

HAL is a multi-disciplinary open access archive for the deposit and dissemination of scientific research documents, whether they are published or not. The documents may come from teaching and research institutions in France or abroad, or from public or private research centers.
L'archive ouverte pluridisciplinaire HAL, est destinée au dépôt et à la diffusion de documents scientifiques de niveau recherche, publiés ou non, émanant des établissements d'enseignement et de recherche français ou étrangers, des laboratoires publics ou privés. 
Florence Lefeuvre

Université Sorbonne Nouvelle - Paris 3 \& CLESTHIA (EA 7345)

Nathalie Rossi-Gensane

Université Lumière Lyon 2 \& ICAR (CNRS UMR 5191)

\section{Les interrogatives indirectes en discours informel oral}

\section{INTRODUCTION}

Les grammaires descriptives de référence (Riegel, Pellat \& Rioul 2009; Wilmet 2003 ; Le Goffic 1993) répertorient essentiellement des interrogatives indirectes (dites encore «percontatives » (Le Goffic 1993 ; Lefeuvre 2006)) du français standard. Les autres types d'interrogatives indirectes sont considérés comme « contraire[s] au bon usage » (Riegel, Pellat \& Rioul, 2009 : 838).

L'objectif de cet article ${ }^{1}$ est d'apporter une analyse descriptive et théorique des interrogatives indirectes totales et partielles en français informel, notamment de voir si les analyses théoriques existantes permettent de mieux comprendre ces interrogatives et, à défaut, de proposer une analyse qui tienne compte de l'ensemble des interrogatives.

S'il existe quelques ouvrages, articles ou chapitres sur l'interrogation indirecte dans un registre informel (v. notamment Defrancq 2000 ; Dagnac 2014), les recherches semblent encore parcellaires ou bien sont centrées sur l'un des français de la francophonie (Ledegen 2007), tant les données de cette sorte ont été peu disponibles jusqu'à encore tout récemment en français hexagonal. La mise en ligne de corpus oraux, comme le CFPP2000 ${ }^{2}$ et les ESLO1- ${ }^{3}$, permet à présent l'accès à une masse importante de données orales plus ou moins informelles. Il est ainsi possible d'avoir un aperçu chiffré des données et même,

1. Nous tenons à remercier les relecteurs anonymes pour leurs remarques.

2. Corpus de Français Parlé Parisien : 684992 mots, soit 49 heures, en mars 2017 (v. Branca-Rosoff et al. 2012).

3. Enquêtes SocioLinguistiques à Orléans : 6,5 millions de mots, soit 508 heures, en 2014. 
grâce à l'intervalle de 40 ans séparant les enregistrements respectifs des ESLO1 et ESLO2, de leur évolution. Nous avons pris pour cette étude le CFPP2000 dans son entier, corpus composé d'interviews conversationnelles, qui permet $\mathrm{d}^{\prime}$ avoir un tableau d'ensemble des interrogatives indirectes introduites par le verbe savoir ${ }^{4}$, verbe le plus fréquemment utilisé à cette fin à l'oral informel (v. Branca \& Lefeuvre 2016). Pour mettre l'accent sur le français oral informel, nous avons sélectionné un sous-corpus des ESLO2, Les repas, genre de discours qui favorise l'émergence de questions et où peut se déployer plus facilement un discours tout à fait libre. Nous avons également prêté attention à un autre verbe, demander, qui n'est pas une forme particulièrement récurrente parmi les verbes introducteurs, mais qui est souvent perçu comme le verbe prototypique pour introduire les interrogatives indirectes (comme le notent par exemple Riegel, Pellat \& Rioul, 2009 : 836).

Nous montrerons que le français informel, présent dans ces corpus de l'oral spontané, produit massivement des interrogatives indirectes de type standard et en beaucoup moins grand nombre, en variation ${ }^{5}$ de ce schéma, des interrogatives indirectes non standard. Ces dernières, peu fréquentes mais diverses de forme, se construisent toutes, si l'on met à part celles introduites pas si, sur le modèle des interrogatives directes. Cela indique, selon nous - et ce sera l'hypothèse principale de cet article -, que la frontière entre les interrogatives directes et les interrogatives indirectes est ténue sur le plan syntaxique, et que les interrogatives indirectes de type non standard se rapprochent de phrases autonomes. On notera d'ailleurs que certaines grammaires adoptent une analyse similaire pour les interrogatives indirectes de type standard. Ainsi, pour P. Le Goffic (1993 : 43), « la subordonnée, enchâssée directement, conserv[e] un caractère proche d'une indépendante ». De manière congruente, pour M. Arrivé, F. Gadet et M. Galmiche (1986 : 354), les interrogatives indirectes sont des subordonnées sans subordonnant, classées à part des conjonctives complétives : «les interrogations partielles ont les mêmes interrogatifs qu'à la forme directe » et si est considéré comme une "transposition de est-ce que ». Toutefois, d'autres grammaires - telle celle de M. Riegel, J.-C. Pellat et R. Rioul (2009) - traitent différemment « interrogatives indirectes totales » et « interrogatives indirectes partielles » :

- les subordonnées interrogatives totales y sont présentées comme étant introduites par « la conjonction si » (ibid. : 838);

- en revanche, certaines subordonnées interrogatives partielles, tout au moins, sont (implicitement) analysées comme des subordonnées sans subordonnant,

\footnotetext{
4. Pour la partie 2 sur les interrogatives indirectes totales, nous nous sommes concentrées sur la forme (ne) sais pas.

5. De nombreux auteurs ont souligné pour la syntaxe (par opposition à la phonologie) le caractère problématique de la notion de variation si celle-ci est définie comme « un changement de forme sans changement de sens » (v. par exemple Boutin \& Rossi-Gensane 2015 pour un rappel de ces discussions). À la suite, notamment, de Gadet $(1997,2010)$, nous considérerons ici comme variantes les constructions concurrentes où la proximité de structures s'accompagne d'une proximité sémantique.
} 
car elles emploient, dans " l'interrogation sur le sujet, l'objet ou l'attribut animés humains » (loc. cit.), le même pronom qui et, dans « l'interrogation sur les circonstances » (ibid. : 839), les mêmes adverbes que l'interrogation directe.

L'interrogation indirecte relevant du discours rapporté, lequel «donne lieu aux mêmes types de phénomènes " dans la mesure où c'est une "pratique sociale dont les enjeux transcendent les langues et les cultures » (Boutin \& Gadet, 2012 : 29), nous esquisserons également, à la faveur de certains exemples, des rapprochements avec les français parlés en Afrique permettant d'envisager, à la suite de B. Boutin et F. Gadet (op. cit. : 31), l'hypothèse d'une " proximité entre vernaculaires d'une même langue ».

La première partie dressera les critères d'identification des interrogatives indirectes $(\S 2)$, la deuxième traitera de certaines interrogatives indirectes totales non standard $(\S 3)$ et la troisième, de certaines interrogatives indirectes partielles non standard (§ 4$)$.

\section{CRITÈRES DE DÉFINITION DES INTERROGATIVES INDIRECTES}

\subsection{Critères de définition}

L'identification des interrogatives indirectes dans les corpus oraux est complexe. Deux grands critères sont concernés :

- Cette identification s'appuie sur un type de verbes susceptibles d'introduire des interrogatives indirectes. Quels sont ces verbes? Ils doivent évoquer un univers de croyance pour R. Martin (1985) et appartenir aux classes larges « savoir » ou « dire » pour G. Serbat (1985 : 9). Selon ces deux auteurs, une donnée doit être problématique ou non expliquée, que ce soit pour le locuteur lui-même ('je ne sais pas') ou pour un autre locuteur ('je sais mais toi peutêtre pas'). P. Le Goffic (1993) propose une liste de 29 verbes susceptibles de régir une interrogative indirecte en complément direct (v. également la liste de Wimmer 1983) : apprendre, comprendre, découvrir, deviner, imaginer, lire, observer, oublier, prévoir, remarquer, savoir, trouver, vérifier, voir, choisir, décider, dire, démontrer, enseigner, expliquer, indiquer, montrer, préciser, raconter, rappeler, révéler, cacher, dissimuler, demander, auxquels est ajouté voilà, " formé anciennement sur l'impératif du verbe voir, [qui] entre encore dans les mêmes constructions que ce verbe » (Le Goffic, 1993 : 181). Pour B. Defrancq (2005 : 170), les interrogatives indirectes " s'associent toutes à des verbes référant d'une façon ou d'une autre à un procès qui a trait à l'information ", mais "l'inverse n'est pas vrai : tous les verbes de ce type ne s'associent pas à une [interrogative enchâssée] ».

- Outre ces verbes introducteurs, il existe des tests syntaxiques proposés par les auteurs (v. pour une synthèse Lefeuvre, 2006 : 149-151) : 
- substitution possible du mot en qu-par comment et quel qui ne peuvent introduire qu'une interrogative indirecte (v. Le Goffic, 1987:84 et Hadermann, 1993 : 126-130) ${ }^{6}$;

- insertion possible de est-ce que dans le segment supposé correspondre à une interrogative indirecte.

\subsection{Recherche des interrogatives indirectes à l'aide des critères de définition}

Voyons comment ces critères s'appliquent dans la recherche des interrogatives indirectes dans nos corpus oraux.

\subsubsection{Type de verbes introducteurs}

La première difficulté pour repérer les interrogatives indirectes dans les corpus oraux est liée au type de verbes introducteurs rencontrés qui n'appartiennent pas toujours à l'une des listes présentées dans les grammaires.

Nous avons ainsi procédé à une étude systématique avec les interrogatives indirectes en quand. Nous avons relevé, dans le CFPP2000, 3369 résultats pour quand, la très grande majorité correspondant au cas où quand est un intégratif (« conjonction » dans Riegel, Pellat \& Rioul 2009). Sur ces 3369 occurrences, nous avons mis en évidence 10 interrogatives indirectes en quand, dont 7 avec un verbe introducteur et 3 se construisant avec n'importe (n'importe quand).

Parmi ces verbes introducteurs, sans surprise, c'est le verbe savoir qui est le verbe introducteur le plus fréquent $(4$ occ.) :

(1) et vot- votre grand-père vous ne savez pas quand i- quand il est mort quand vo- (CFPP2000, 20-01)

Ensuite, nous avons trouvé le verbe se souvenir relevant du champ lexical de la mémoire, régissant un complément indirect, et non un complément direct (Wimmer 1983 le signale bien dans sa liste, ainsi que Riegel, Pellat \& Rioul 2009) :

(2) et: et euh:: je je je me souviens quand il m'avait interrogé (CFPP2000, 12-01) Enfin, nous avons pu relever le verbe demander (1 occ.) :

(3) je n'vais pas demander à [Mathieu] quand il est arrivé dans l'quartier parce que je l'sais (CFPP2000, 12-04)

D'autres structures rencontrées sont sujettes à discussion. En premier lieu, plusieurs constructions n'appartiennent pas aux listes habituelles fournies dans les grammaires consacrées au français standard ; c'est le cas de :

6. Nous ne prenons pas en compte ici les subordonnées en quel que soit. 
- des questions sur:

(4) donc mes mes premières questions sont des questions tout à fait traditionnelles sur depuis quand vous êtes arrivée dans le quartier (CFPP2000, 14-01)

- être au courant de :

(5) je lui ai dit "ah vous êtes au courant + quand est-ce que c'est l'assemblée de copropriétaires ?" (CFPP2000, 05-01)

Dans ce dernier cas, l'exemple contenant être au courant pourrait se paraphraser à l'aide du verbe savoir :

(6) “ah vous $\{\hat{e} t e s$ au courant / savez $\}$ + quand est-ce que c'est l'assemblée de copropriétaires ?"

\subsubsection{Tests syntaxiques : insertion de « est-ce que ", substitution de} " qu- "

La présence de est-ce que dans la subordonnée (5)-(6) ainsi que la possibilité de changer le mot en qu- (7) montrent que les tests syntaxiques répondent positivement :

(7) a. "ah vous êtes au courant + comment a été mise en place l'assemblée de copropriétaires?"

b. "ah vous êtes au courant + à quelle heure est la réunion ?"

Cela dit, la pause permet également d'avoir une tout autre lecture de cet énoncé et d'y voir deux phrases : vous êtes au courant et à quelle heure est la réunion?.

L'exemple (4) réunit deux prépositions, sur et depuis, l'une dépendant de la structure de la principale (des questions sur) et l'autre impliquée par la subordonnée, où elle introduit un circonstant temporel. Cette structure répond positivement aux tests :

(8) a. donc mes mes premières questions sont des questions tout à fait traditionnelles sur depuis quand est-ce que vous êtes arrivée dans le quartier

b. donc mes mes premières questions sont des questions tout à fait traditionnelles sur dans quel quartier vous avez habité

Elle est interprétée comme une tournure percontative par les locuteurs, comme le montre l'enchaînement de ces tours de parole :

(9) spk1 : donc mes mes premières questions sont des questions tout à fait traditionnelles sur depuis quand vous êtes arrivée dans le quartier spk2 : euh alors ben parce que moi moi finalement j'ai un peu j'ai un peu parcouru l'quatorzième (CFPP2000, 14-01)

Selon une autre analyse, nous pourrions voir dans cet énoncé deux phrases, une assertive interrompue (donc mes mes premières questions sont des questions tout à fait traditionnelles sur) et une interrogative (directe) (depuis quand vous êtes arrivée dans le quartier). Cependant, rien ni dans la pause ni dans le contour mélodique de cet 
ensemble n'indique qu'il s'agit d'une interrogative directe. Les deux structures de phrase sont liées et aucun accent ne porte sur depuis quand. Nous verrons donc bien une percontative dans cet énoncé.

\subsubsection{Une structure interrogative?}

Une autre difficulté dans la recherche des interrogatives indirectes concerne les structures amorcées par un mot en qu-qui peuvent en fait ne pas correspondre à des interrogatives, mais à des conjonctives. Ainsi, dans cet exemple :

(10) puis après on $\mathrm{a}+\mathrm{euh}+$ je me rappelle plus quand on était à Bois-Colombes si on allait beaucoup au cinéma ou pas (CFPP2000, 05-01)

malgré la présence d'un verbe introducteur (se rappeler), quand on était à BoisColombes n'est pas régi comme un complément essentiel direct par me rappelle, mais est un circonstant. C'est si on allait beaucoup au cinéma ou pas qui est l'interrogative indirecte régie par ce verbe ${ }^{7}$.

Ce cas de figure peut intervenir également lorsque le verbe introducteur est employé de façon absolue. Ainsi en est-il de demander dans l'exemple suivant qui signifie ici 'poser des questions' :

spk1 : vous n'en aurez plus [de chien] ?

spk2 : ben + les gens m'ont beaucoup demandé quand il est mort parce que ça a été beaucoup me disaient alors bon et en reprendre un je disais (CFPP2000,14-01)

Les verbes introducteurs types peuvent apparaître dans un tout autre emploi. C'est le cas de savoir susceptible d'être utilisé comme marqueur discursif :

(12) mais ça n'a pas continué parce que c'est $(\mathrm{mm})$ alors c'est toujours pareil quand vous savez quand il faut préparer $+(\mathrm{mm})$ la fête d'Ivry qu'il faut pmoi j'étais complètement débordée donc (CFPP2000 IV-01)

Vu les résultats de cette étude et la difficulté à repérer les interrogatives indirectes, nous avons choisi de nous en tenir à des types de verbes pouvant introduire en nombre suffisamment élevé des interrogatives indirectes. Le verbe savoir, à l'oral spontané, est le verbe le plus employé dans cette configuration. Nous avons pris également un autre verbe, demander, qui est le verbe généralement considéré comme prototypique dans un tel rôle (v. par exemple Riegel, Pellat \& Rioul, 2009: 836).

7. Signalons que, si la subordonnée conjonctive circonstancielle introduite par quand est incidente, sur un plan syntaxique, du fait de sa position, à la proposition principale, elle porte néanmoins sémantiquement sur l'interrogative indirecte. 


\section{LES INTERROGATIVES INDIRECTES TOTALES}

Pour M. Arrivé, F. Gadet et M. Galmiche (1986 : 354), « le seul type d'interrogation [indirecte] totale possible est la transposition de est-ce que en si », la conservation de est-ce que n'étant évoquée qu'en rapport avec l'interrogation indirecte partielle. De même, M. Riegel, J.-C. Pellat et R. Rioul (2009 : 838) considèrent que « la seule structure possible utilise la conjonction si pour introduire la subordonnée interrogative, à l'oral comme à l'écrit [...]. Il n'y a ni inversion, ni possibilité d'utiliser est-ce que ».

Dans le prolongement de ces remarques, l'interrogation indirecte totale ne connaîtrait pas de variation. L'exemple suivant met, à cet égard, en contraste interrogation indirecte totale, réalisée de manière standard, et interrogation indirecte partielle, réalisée de manière non standard :

(13) et puis j'allais pas leur demander si elles allaient monter ou descen- euh ou qu'est-ce qu'elles faisaient (CFPP2000, SO-01)

Les interrogatives partielles admettent de fait des variantes diversifiées (cf. § 4 infra), d'ailleurs déjà en beaucoup plus grand nombre dans l'interrogation directe que les interrogatives totales. Ainsi, A. Coveney (2011 : 114), parmi d'autres, mentionne dix sortes de structures interrogatives directes partielles contre quatre sortes de structures interrogatives directes totales, lesquelles s'appuient sur une intonation montante (uniquement), sur la présence de est-ce que ou, enfin, sur un ordre particulier, telles ladite « inversion du clitique » et ladite « inversion complexe » (que l'on trouve par exemple dans Les enfants viennent-ils?).

En relation avec ce qui précède, on note que, dans les ESLO1-2 comme dans le CFPP2000, les interrogatives totales (tout au moins après le verbe demander et après la forme (ne) sais pas sur laquelle nous nous concentrons dans cette partie) se réalisent dans une immense majorité de manière standard. Ainsi :

- dans les ESLO2_Les repas, on compte 9 interrogatives totales après le verbe demander et 18 après la forme (ne) sais pas, toutes normées (mais parfois interrompues) sauf une après le verbe demander que l'on peut considérer comme non normée du fait de la non-transposition des temps ;

- dans le CFPP2000, le verbe demander est suivi de 16 interrogatives totales normées (parfois interrompues), d'une interrogative totale non normée apparaissant dans une liste et de 4 interrogatives totales que l'on peut considérer comme non normées du fait de la non-transposition des temps; la forme (ne) sais pas est suivie de 111 interrogatives totales normées (parfois interrompues), de 2 interrogatives totales non normées apparaissant dans une liste et d'une interrogative totale non normée (voire une seconde, moins décidable) en raison de l'absence de si.

Dans ce qui suit, en recourant éventuellement aussi à des exemples tirés de l'intégralité du corpus des ESLO1-2, nous présenterons quelques cas d'interrogatives indirectes non standard stricto sensu, en relation avec le phénomène de 
la prolepse, l'absence de si, la coexistence entre que et est-ce que et la présence de est-ce que dans des listes et, lato sensu, en relation avec la non-transposition des temps et la présence, notamment, de " ligateurs énonciatifs » (Morel \& DanonBoileau 1998) dans le voisinage de si. Nous évoquerons également, à la marge, le procédé particulier de postposition de la séquence je (ne) sais pas à une interrogative totale introduite par est-ce que.

\subsection{Les interrogatives indirectes totales non standard en si}

\subsubsection{De la prolepse}

M. Riegel, J.-C. Pellat et R. Rioul (2009 : 838) mentionnent, pour l'interrogation totale, une (seule) possibilité d' « écart » par rapport au standard, attribuée à la langue familière, qui consiste à « anticip[er] (par prolepse) comme complément d'objet direct du verbe principal » le syntagme nominal de la subordonnée « qui a la valeur thématique la plus saillante ». Le corpus des ESLO1-2 en comporte un seul exemple après la forme (ne) sais pas :

(14) ah je sais pas le tennis si c'est pareil que parce que par exemple au volley si tu as pas ta licence $\mathrm{hm}$ eh ben tu peux payer aussi (ESLO2_REPAS_1267_C)

\subsubsection{De la non-transposition des temps}

Dans certains exemples, l'interrogative indirecte introduite par si ne comporte pas de transposition des temps. Cette non-transposition est facilitée dans l'exemple suivant par la présence $d^{\prime}$ une parenthèse :

(15) spk1 : oui + je vous demandais ça parce que je m'demandais si euh à Paris on + on était les amis enfin vous c'est différent vous avez tout c'milieu littéraire qui a

spk2 : oui

spk1 : et puis après l'milieu de l'ADAC euh mais si on a un noyau d'amis qui s'constitue au moment où on est dans son réseau euh de classe quoi de lycée (CFPP2000, 07-03)

Il est intéressant de préciser que l'absence de transposition, notamment des temps, dans le discours indirect a beaucoup été signalée comme une tendance des français parlés en Afrique (v. par exemple Ngamountsika, 2013 : 129, 136141).

\subsubsection{Vers un rôle de balise pour « si ॥ ?}

Participe également d'un brouillage de la distinction entre discours indirect et discours direct - L. Rosier (1999 : 202) parle à cet égard de formes «mixtes » ou de formes « hybrides »- le fait que, dans certains exemples, si introducteur de discours indirect soit immédiatement précédé ou suivi d'éléments tels que bon, ben, oui rangés par M.-A. Morel et L. Danon-Boileau (1998) dans les « ligateurs énonciatifs » (on compte trois cas dans les ESLO pour bon ou ben et un cas dans le CFPP2000 pour oui), ou encore l'interjection oh « marqueur de la surprise et de la relation à soi » (ibid. : 97) : 
(16)

et je voulais savoir bon si elles sont importantes pour vous si vous les suivez ou pas (ESLO2_ENT_1077)

euh le plus pour moi c'est d'avoir du travail du travail qui me plaît et si mes chefs sont contents euh de leur demander ben si je peux avoir un petit avancement (ESLO1_ENT_087)

spk1 : j'me demandais $s i+o u i$ les boubous les j'sais pas quoi les

spk1 spk4 : [1] foulards + [2] ah pas du tout

spk1 : palestiniens

spk4 spk1 : [1] ah pas du tout [2] les + (CFPP2000, 11-03)

euh déjà il accepte beaucoup de mots qui sont pas enfin il faut bien en ajouter il faut bien en enlever mais enfin je sais pas oh si vraiment $c^{\prime}$ est très utile (ESLO1_ENT_150)

Or, selon H. L. Andersen (2000 : 148), les « marqueurs [tels] bon, bon ben, alors, tiens, écoute, euh, tu sais, franchement, mais, un prénom (vocatif) ou des exclamations [comme] oh là là » indiquent le début du discours direct. L. Rosier (2008 : 78) évoque des « discordanciels [...] permett[a]nt de produire un décrochage énonciatif ». Sont notamment mentionnées les « interjections » telles que $a h$, eh bien, etc. qui «tendent également le discours vers le direct de l'énonciation » (ibid. : 80). En outre, on notera, dans l'exemple 16, une éventuelle non-transposition des temps ${ }^{8}$ (sans que l'on puisse se prononcer sur l'exemple 18 où l'interrogative, inachevée, ne comporte pas de verbe).

Il a été parfois signalé, pour les français parlés en Afrique (v. par exemple Boutin \& Rossi-Gensane 2015), un rôle d'organisation du discours de que (outre son rôle syntaxique de subordonnant ouvrant une conjonctive complétive) de par sa contiguïté avec des ligateurs énonciatifs qui « pourrait être une motivation à la réanalyse de que comme balise introductrice et décrocheur énonciatif » (ibid. : 172) :

vers la fin il me dit que non mon papier il n'a pas pu prendre que c'est trop compliqué que lui il pensait que c'était un autre travail et moi je lui ai ddonné un travail que son ami ne peut pas faire que c'est trop pour son ami (Abidjan in Boutin 2011a ; citée par Boutin \& Rossi-Gensane, 2015 : 172)

le gars il dit non que non que le que l'onc- que Monsieur euh Kaboré qu'il est sorti (Ouagadougou in Prignitz 2007 ; citée par Boutin \& Rossi-Gensane, 2015 : 172)

8. Conformément à ce que suggère l'un de nos relecteurs, l'imparfait dans la proposition principale, plutôt que doté de la valeur du passé, peut être interprété ici comme « une demande polie » (Riegel, Pellat \& Rioul, 2009 : 547). 
De même, la contiguïté entre si et des ligateurs énonciatifs dans des occurrences telles que (16)-(19) pourrait permettre d'envisager d'étendre à ce premier élément, qu'on le considère, ou non, comme un subordonnant, ce rôle de balise de discours, qui plus est en français hexagonal ${ }^{9}$.

\subsubsection{De l'absence de " si »}

On note quelques rares cas où une interrogative indirecte est produite après la forme sais pas en l'absence de si :

(22) ça c'est mon point de vue je sais pas je me trompe l'avenir me dira si je me trompe (ESLO1_INTPERS_438)

(23) y a un petit château avec un parc un petit sorte de lac j'sais pas c'est un lac artificiel (CFPP2000, SO-02)

Pour ce dernier exemple, il est également intéressant de noter que, par la suite, le locuteur réitère la même interrogative, cette fois-ci en l'introduisant par si :

$$
\begin{aligned}
& \text { spk4 : y a un sor- y a un château euh } \\
& \text { spk1 : près d'l'au-X parc } \\
& \text { spk4 : très beau et y a un petit lac [ouais] je sais pas si c'est un lac artificiel } \\
& \text { spk1 : oui (CFPP2000, SO-02) }
\end{aligned}
$$

Cette absence de si n'est pas sans évoquer l'existence, signalée, parmi d'autres, par L. Rosier (2008: 98), d'un discours indirect sans que, qui « montrerait que le marqueur de subordination n'est pas inhérent à la catégorie de l'indirect ». À la différence de ce qu'affichent les exemples supra, l'absence de que dans de tels cas est volontiers " compensée », en quelque sorte, par la présence de ligateurs énonciatifs assurant alors le balisage (v. aussi Ngamountsika, 2013 : 144, pour d'autres exemples en français parlé hexagonal et dans les français parlés en Afrique) :

(25) il dit bon euh, l'embellie est finie pour le moment (Liège in Hambye \& Simon 2006 ; cités par Boutin \& Rossi-Gensane, 2015 : 172)

(26) Fabienne me dit ben euh, c'est bien la preuve de, de (Liège in Hambye \& Simon 2006 ; cités par Boutin \& Rossi-Gensane, 2015 : 172)

(27) il me dit qu'est-ce qui se passe je dis non je viens de la Justice ils m'ont donné un délai qui ne m'arrange pas tout ça (Abidjan in Boutin 2011a ; citée par Boutin \& Rossi-Gensane, $2015:$ 172)

9. À ce propos, Boutin \& Rossi-Gensane (2015 : 173) notent que «l'entrée de que dans l'inventaire des balises introductrices de discours n'a pas fait l'objet d'études hors de l'Afrique [...] alors que le phénomène ne se limite probablement pas à ce continent ». 


\subsection{Les interrogatives indirectes totales non standard en est-ce que}

Quelques interrogatives totales marquées par est-ce que apparaissent dans un contexte de discours rapporté indirect. Ces occurrences semblent soumises à une condition de non-contiguïté entre verbe introducteur et est-ce que, telles la présence d'une subordonnée qui s'intercale entre ce verbe introducteur et l'interrogative totale, ou encore l'appartenance de l'interrogative totale à une liste dont elle n'est pas le premier élément.

\subsubsection{De la coexistence entre " que " et " est-ce que "}

L'occurrence suivante a été produite par une locutrice née en Martinique, mais arrivée à Orléans l'année de sa naissance ${ }^{10}$ :

euh voilà là on a demandé que bon c'est vrai que c'est c'est démoli mais on a demandé que quand ils vont reconstruire est-ce qu'ils peu-i- de mettre une petite stèle une petite pierre (ESLO2_ENT_1083_C)

Dans cet exemple, que ne peut être vu comme un subordonnant conjonctif que si l'on admet soit une sorte de cumul entre conjonctive complétive et interrogative indirecte, soit une sorte d'enchâssement dans la conjonctive complétive de l'interrogative indirecte (celle-ci étant cependant abandonnée après les amorces peu- et $i$-). On peut aussi choisir d'attribuer un rôle purement démarcatif à que, qui, en fait, n'ouvre pas ici une " assertion indirecte ». À cet égard, alors que l'on ne peut se prononcer sur une transposition ou pas des pronoms (ils), on notera, dans cette séquence introduite par que, l'absence de transposition des temps (vont reconstruire, amorce verbale peu-) susceptible de questionner son intégration syntaxique. Par ailleurs, la présence de la conjonctive circonstancielle introduite par quand paraît faciliter l'apparition de l'interrogative indirecte en estce que en s'interposant entre que et cette dernière (alors que, de manière standard, la conjonctive circonstancielle devrait être incluse dans l'interrogative indirecte). Un parallèle peut être établi avec certains exemples dans des français parlés en Afrique :

(29) je lui ai dit que bon si j'amène la moto ici est ce tu peux acheter (Queffélec 2006 ; cité par Dagnac 2014)

Ainsi, A. Dagnac (2014) signale un phénomène « particulièrement [...] étudié pour le Cameroun [où] que semble intonativement rattaché au verbe introducteur et associé à un intonème montant [et] peut [notamment] être suivi d'un interrogatif en est-ce que ».

À propos des relatives non standard, F. Gadet (1989 : 154) remarque de même, dans certains cas, des « incises de natures assez variées » (toutefois majoritairement des conjonctives circonstancielles) immédiatement après le que, de sorte

10. Néanmoins, on ne connaît pas l'importance des interactions entre la locutrice et les membres d'origine martiniquaise de sa famille, ce qui ne permet pas d'appréhender la part d'éventuels facteurs diatopiques. 
que l'incise " constitu[e] une sorte d'écran » et/ou «fait perdre le fil de la relative au locuteur et [...] est suivie d'une phrase indépendante (cependant, [sans $\mathrm{qu}^{\prime}$ ]aucune de ces phrases ne comporte d'intonation suspensive) ».

\subsubsection{Des listes}

L'apparition d'une interrogative totale marquée par est-ce que dans un contexte de discours rapporté indirect semble également favorisée par la présence de cette interrogative dans une liste dont elle n'est pas le premier élément. Ainsi, dans l'exemple suivant, pour lequel on ne peut se prononcer sur une transposition, ou pas, des pronoms et des temps (du fait, respectivement, de l'utilisation, d'une part, d'un vous générique et, d'autre part, du présent dans le discours citant et dans le discours cité), les deux interrogatives totales non standard suivent une interrogative partielle standard :

(30) on vous demande des + depuis combien d'temps vous travaillez $+(\mathrm{mm})$ est-ce que vous êtes en contrat à durée indéterminée + XX XX est-ce que vous avez un emploi stable $(\mathrm{mm})+$ eh ben non (CFPP2000, Ivry 01)

On signalera toutefois une intonation qui, peut-être en raison d'un " format liste », semble intermédiaire entre discours direct et discours indirect, ainsi que $\mathrm{d}^{\prime}$ assez longues pauses entre les questions.

\subsection{3. À la marge : interrogative totale introduite par " est-ce que " et postposition de la séquence " je (ne) sais pas "}

Il est parfois recouru à un procédé particulier d'interrogation totale en relation avec la séquence je (ne) sais pas, qui est alors postposée à une interrogative introduite par est-ce que (on compte 7 cas dans le CFPP2000) :

alors est-ce que c'est bien je sais pas (CFPP2000, 15-02)

alors est-ce qu'il allait parler je sais pas (CFPP2000, 13-04)

Au premier abord, on pourrait être tenté d'évoquer, par certains côtés, un emploi parenthétique de je (ne) sais pas ${ }^{11}$, si l'on met en correspondance ces dernières occurrences avec, respectivement :

$$
\begin{aligned}
& \text { je sais pas si c'est bien } \\
& \text { je sais pas s'il allait parler }
\end{aligned}
$$

On note d'ailleurs un cas de coexistence entre si et est-ce que, contiguë et sans pause (mais sans doute avec un léger accent sur est-ce que) dans un exemple " encadré " par je (ne) sais pas, où si peut être vu comme rattaché à gauche et est-ce que, à droite :

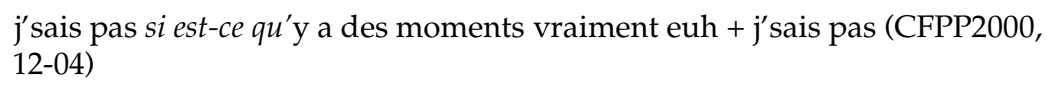

j'sais pas si est-ce qu'y a des moments vraiment euh + j'sais pas (CFPP2000, 12-04)

11. Schneider (2007 : 79-80) inclut je sais pas dans les verbes parenthétiques, comme le signale également Gachet (2015: 201). 
Selon F. Gachet (2015: 319), « un verbe parenthétique se définit par la possibilité $\mathrm{d}$ 'entrer dans deux constructions, en position initiale devant une que-P[hrase] ou en position d'incise finale postfocale ». Néanmoins, dans de tels cas, la séquence je (ne) sais pas, outre le fait qu'en position initiale, elle ne serait pas suivie par une que-P[hrase] mais par une "si-Phrase », constitue un nouvel énoncé qui « se prononce avec l'intonation des phrases indépendantes » (Vet, 1994 : 59). Dans les termes de la macrosyntaxe aixoise, on a donc affaire non à un noyau et à un postnoyau, mais à deux noyaux possédant d'ailleurs chacun une force illocutoire de nature différente, respectivement interrogative et déclarative : une question et sa réponse, donnée par le même locuteur.

La séquence je (ne) sais pas ne peut notamment pas prétendre à être une " incise de discours rapporté ", car celle-ci "ne peut pas contenir de renvoi anaphorique au contenu des paroles rapportées » (Gachet, $2015: 100){ }^{12}$ comme c'est le cas dans l'exemple suivant sous la forme du pronom personnel le :

(36) est-ce qu'on pourra faire ce qui était prévu je ne le sais pas en tout cas il y a déjà pas mal de réalisations (ESLO1_ENT_078_C)

Cependant, bien que la séquence je (ne) sais pas constitue un énoncé (un noyau), elle se caractérise, entre autres, par un blocage de sa polarité, propre, selon C. Blanche-Benveniste (1989), aux verbes parenthétiques, qu'elle appelle recteurs faibles. En effet, comme le note C. Vet (1994:60), on peut répondre à une question par je (ne) sais pas, mais pas par je sais :

$$
\text { *alors est-ce que c'est bien je sais (d'après l'exemple 32) }
$$

La séquence je (ne) sais pas - à la fois forme épistémique, notamment à la première personne du présent de l'indicatif ${ }^{13}$, et (par une sorte de dérivation sémantique, le constat d'ignorance appelant question) forme de discours rapporté dans une construction avec interrogative indirecte (mais pas verbe de discours rapporté direct) - participe ainsi d'une routinisation permettant de formuler en premier l'interrogative totale. Des études ultérieures, fondées sur des corpus plus étendus, devront confirmer, ou non, la productivité de ce procédé très à la marge de l'interrogation indirecte.

12. Gachet $(2015: 295)$ précise : «Cette caractéristique semble s'appliquer à tous les parenthétiques. Une séquence comme je le crois, il me le semble, il me dit ça, j'ai vu ça ne peut pas se trouver en position d'incise finale ; sa réalisation prosodique en fait au contraire un nouvel énoncé ».

13. Le statut épistémique de je (ne) sais pas est néanmoins mentionné par Vet (1994: 58) comme étant à part, car « [i]l n'y a pas, dans le domaine de connaissances, de compartiment qui porte l'étiquette <Je ne sais pas $>$ ». 


\section{LES INTERROGATIVES INDIRECTES PARTIELLES}

\subsection{Méthodologie et résultats bruts}

Pour le CFPP2000, nous avons utilisé des expressions régulières permettant de rechercher les formes du verbe savoir avec les différents interrogatifs ${ }^{14}$. Les résultats bruts du CFPP2000 apparaissent dans le tableau infra pour savoir, avec 231 interrogatives indirectes relevées, dont 144 avec un verbe conjugué.

Tableau 1 : Savoir et les interrogatives indirectes dans le CFPP2000 a

\begin{tabular}{|c|c|c|c|c|c|c|c|c|c|}
\hline $\begin{array}{l}\text { Int. ind. } \\
\text { en qu- }\end{array}$ & Total & SV & VS & $\begin{array}{c}\text { Est-ce } \\
\text { que }\end{array}$ & $\begin{array}{l}\text { Qu- } \\
\text { que }\end{array}$ & $\begin{array}{l}\text { In } \\
\text { situ }\end{array}$ & Vinf & $\begin{array}{l}\text { Seul mot } \\
\text { en qu- }\end{array}$ & Av. \\
\hline où & 44 & 19 & 2 & 6 & 0 & 0 & 7 & 10 & 0 \\
\hline quand & 7 & 5 & 0 & 1 & 0 & 0 & 1 & 0 & 0 \\
\hline comment & 72 & 36 & 2 & 0 & 2 & 0 & 26 & 6 & 0 \\
\hline pourquoi & 26 & 12 & 0 & 0 & 0 & 0 & 0 & 14 & 0 \\
\hline combien & 3 & 0 & 0 & 1 & 0 & 0 & 0 & 1 & 1 \\
\hline quel & 15 & 7 & 0 & 0 & 0 & 0 & 2 & 5 & 1 \\
\hline qui & 19 & 12 & 3 & 2 & 0 & 0 & 1 & 1 & 0 \\
\hline quoi & 13 & 1 & 0 & 0 & 0 & 1 & 4 & 7 & 0 \\
\hline ce que & 28 & 28 & 0 & 0 & 0 & 0 & 0 & 0 & 0 \\
\hline qu'est-ce qu- & 4 & 0 & 0 & 4 & 0 & 0 & 0 & 0 & 0 \\
\hline Total & 231 & 120 & 7 & 14 & 2 & 1 & 41 & 44 & 2 \\
\hline & $100 \%$ & \multicolumn{5}{|c|}{144 int. ind. à V conjugué (62 \%) } & $18 \%$ & $19 \%$ & $1 \%$ \\
\hline
\end{tabular}

a. Int. ind. = Interrogatives indirectes $; S=$ Sujet ; V = Verbe ; Vinf = Verbe à l'infinitif ; Av. = Averbales

En revanche, avec le verbe demander, les résultats sont au nombre de 12 : demander ce que (2), demander où (1), demander comment (6), demander quel (2), demander pourquoi (1) :

(38) ben justement on se demande comment on a pu vivre avant sans les supermarchés ++ (CFPP2000, MO-03)

Pour le sous-corpus des ESLO2_Les repas, nous n'avons pu établir un comptage qu'à partir d'une méthode artisanale en relevant systématiquement les occurrences de toutes les formes du verbe savoir. Nous avons ainsi répertorié 98 interrogatives indirectes à verbe conjugué.

14. Merci à Serge Fleury pour son aide dans l'écriture de ces expressions. 
Les interrogatives indirectes en discours informel oral

Tableau 2 : Savoir et les interrogatives indirectes dans les ESLO2_Les repas a

\begin{tabular}{|c|c|c|c|c|c|c|}
\hline Mots en qu- Int. Ind. & Total & SV & VS & Est-ce que & Qu-que & In situ \\
\hline où & 15 & 13 & 1 & 0 & 1 & 0 \\
\hline quand & 2 & 0 & 0 & 1 & 0 & 1 \\
\hline comment & 17 & 15 & 1 & 0 & 1 & 0 \\
\hline pourquoi & 4 & 4 & 0 & 0 & 0 & 0 \\
\hline combien & 5 & 5 & 0 & 1 & 0 & 0 \\
\hline quel & 2 & 2 & 0 & 0 & 0 & 0 \\
\hline lequel & 1 & 1 & 0 & 0 & 0 & 0 \\
\hline qui & 13 & 12 & 0 & 0 & 0 & 1 \\
\hline quoi & 4 & 1 & 0 & 0 & 0 & 3 \\
\hline ce que & 34 & 34 & 0 & 0 & 0 & 0 \\
\hline qu'est-ce qu- & 1 & 0 & 0 & 1 & 0 & 0 \\
\hline Total & 98 & 87 & 2 & 2 & 2 & 5 \\
\hline & $100 \%$ & \multicolumn{5}{|c|}{98 int. ind. à $\mathrm{V}$ conjugué } \\
\hline
\end{tabular}

a. Int. ind. = Interrogatives indirectes ; S = Sujet ; $V=$ Verbe

Avec le verbe demander, nous avons relevé 6 occurrences : demander à quoi (1), demander combien (1), demander comment (2), demander pourquoi (1), demander où (1) :

\subsection{Les interrogatives indirectes partielles standard}

D'après le tableau 1 supra pour le CFPP2000, les interrogatives indirectes partielles se répartissent en trois grands groupes: les interrogatives indirectes basées sur un verbe conjugué, celles qui comprennent un infinitif et celles qui se réduisent au mot en $q u$-. Les interrogatives indirectes qui s'appuient sur un verbe conjugué sont massivement représentées dans nos deux corpus, le CFPP2000 (120) et le sous-corpus des ESLO2_Les repas (87), selon le schéma du type $<$ mot en $q u$-SV $>$, avec une faible variante en VS (pour savoir, 7 exemples pour le CFPP2000 et 2 pour les ESLO2_Les repas). Nous obtenons $88 \%$ de ce type d'interrogatives indirectes pour le CFPP2000 et 90 \% pour les ESLO2_Les repas.

Pour les interrogatives indirectes qui suivent le schéma VS :

(40) mais ils savent pas où est le nord (CFPP2000, 15-01)

(41) surtout quand on sait comment fonctionnent un petit peu les prix littéraires (CFPP2000, 14-01)

(42) et tu sais qui est Philippe Auguste et qui est Saint Louis ? (CFPP2000, 12-04) 
la postposition du sujet est en lien

[avec] la fonction du connecteur dans la subordonnée : plus cette fonction est étroitement liée à la relation prédicative, plus la place immédiatement postérieure au verbe est laissée libre pour une postposition éventuelle du sujet nominal. (Le Querler, 1997 : 180)

\subsection{Les interrogatives indirectes partielles non standard}

Le premier constat qui s'impose est que les interrogatives indirectes partielles non standard représentent un pourcentage peu élevé (12\% pour le CFPP2000 et $10 \%$ pour les ESLO2_Les repas). Trois types figurent dans ces corpus.

\subsubsection{Les interrogatives indirectes partielles en " est-ce que "}

Les interrogatives indirectes partielles en est-ce que sont au nombre de 14 avec les formes du verbe savoir dans le CFPP2000 (et 21 en tout, quel que soit le verbe introducteur) :

(43) je je je sais pas où est-ce que je vais (CFPP2000, 07-04)

(44) alors moi j'sais même plus quand est-ce qu'il est devenu à la mode (CFPP2000, 11-01)

(45) je sais pas combien est-ce que c'était chaque trois + semaines ou chaque trois mois je me rappelle plus (CFPP2000, 14-02)

(46) mais euh pour savoir qui est-ce qui qui est-ce qu'on allait avoir (CFPP2000, 07-02)

(47) tout l'monde sait qu'est-ce que vous avez si vous si vous manquez d'argent si vous avez pas d'travail (CFPP2000, MO-03)

Signalons également l'emploi de comment avec un autre verbe introducteur :

$$
\text { mais j'attends de voir comment est-ce qu'elle évolue (CFPP2000, 13-02) }
$$

Des mots en qu- autres que qui et que peuvent donc introduire ces interrogatives indirectes (notamment où et quand dans le CFPP2000), à l'encontre de ce qu'avance B. Defrancq (2000 : 135).

Si les spécialistes de l'oral spontané relèvent ce type de structure (v. Defrancq, $2000: 134-135)$, les grammaires descriptives de référence ne le citent qu'en marge (v. Riegel, Pellat \& Rioul, 2009: 839 dans une remarque), sans le décrire ni l'expliquer :

Le français populaire a suivi sa propre logique : Dis-moi qu'est-ce qui te gêne / qu'est-ce que tu veux. (Wilmet, $2003: 285$ )

Aux $\mathrm{XVII}^{\mathrm{e}}$ et $\mathrm{XVIII}{ }^{\mathrm{e}}$ siècles, ces interrogatives indirectes appartenaient au français standard (v. Lefeuvre 2014). Elles pouvaient figurer dans toutes sortes d'écrits, des mémoires :

(49) me demandant qu'est-ce que c'était que des tambours qui battaient (Cardinal de Retz ; cité par Lefeuvre 2014) 
une lettre de Fénelon au Roi :

(50) Vous demanderez peut-être, sire, qu'est-ce qu'ils doivent vous dire ; le voici : ils doivent vous représenter qu'il faut vous humilier. (Fénelon, Lettre à Louis XIV 1693 - Frantext)

dans les Salons rédigés par Diderot :

(51) On demandera à jamais qui est-ce qui disposait des marbres du souverain. À la place du Marigny, j'entendrais sans cesse cette question et je rougirais. (Diderot, Salon de 1767)

Elles n'étaient pas uniquement réservées aux personnages représentant le peuple, même si c'était possible :

Il faut voir de quoi est-ce qu'elle est malade. (Molière, Le médecin malgré lui, 1667)

La présence de est-ce que dans les interrogatives indirectes permet de proposer l'hypothèse syntaxique suivante : ces structures construiraient leur enchâssement à une structure de phrase non pas par un subordonnant en $q u$ - mais en discours sans subordonnant proprement dit ; $d$ 'autres facteurs (contexte, prosodie) indiqueraient l'enchâssement d'une sous-phrase dans une phrase. Nous nous rapprochons ainsi de ce qui est suggéré pour « l'usage familier » dans la grammaire de M. Riegel, J.-C. Pellat et R. Rioul (2009) :

L'introduction du marqueur interrogatif est-ce que / qui crée un effet de juxtaposition syntaxique qui aligne la structure indirecte sur le modèle de l'interrogation directe indépendante. (Riegel, Pellat \& Rioul, 2009 : 839)

On peut se demander, dès lors, si les interrogatives indirectes de type standard ne construiraient pas de la même façon leur lien de subordination : " la subordonnée, enchâssée directement, conserv[e] un caractère proche d'une indépendante » (Le Goffic, 1993 : 43).

Cela dit, est-ce que peut se trouver également dans des relatives :

ah ++ avant + pas celle où est-ce que où est-ce qu'elle avait MJC (CFPP2000, SO-02)

ou figurer là où l'on attendrait une relative périphrastique en ce que / ce qui :

je pensais plutôt à qu'est-ce qui se passe dans la cour de récréation (CFPP2000, 11-02)

Il est intéressant de signaler la présence dans les ESLO1 de propositions subordonnées relatives introduites par qui est-ce qui :

(56) c'est des voitures qui est-ce qui reviennent le moins cher je crois (ESLO1_ENT_111) 
et là j'en ai y en a cinq qui est-ce qui appartiennent à mon fils (ESLO1_ENT_045)

Il semble que l'on ait, dans ce dernier cas, affaire à un phénomène pour lequel jouent des facteurs diachroniques et, sans doute, diastratiques. Ces relatives sont en effet produites par des locuteurs nés dans les années 1900 ou 1910 et ayant quitté l'école entre 11 et 14 ans.

Dans les propositions relatives en est-ce que (dont le caractère enchâssé ne peut être mis en doute), on pourrait voir un subordonnant complexe du type mot en qu- suivi de est-ce que, celui-ci se fondant dans le mot en qu-relatif. Une autre possibilité, dans le prolongement de ce qui précède, serait d'analyser ces structures comme des groupes autonomes, mais enchâssés grâce à d'autres paramètres qui seraient ici la présence d'un antécédent (celle dans l'exemple 54) ou d'une préposition (à dans l'exemple 55).

Notons également que notre sous-corpus des ESLO2 ne contient presque pas d'interrogatives indirectes partielles en est-ce que ( 2 , ce qui donne $2 \%$ vs 9,7\% pour le CFPP2000 avec les formes de savoir). Ce qui peut expliquer ce résultat, $c^{\prime}$ est la présence d'un autre type de percontative, in situ, qui prendrait la place de ce type de structure (cf. § 4.3.3).

\subsubsection{Les interrogatives indirectes partielles en "qu- que "}

Nous avons répertorié deux interrogatives indirectes en qu-que dans le CFPP2000 introduites par une forme du verbe savoir :

(58) je sais pas comment que ça se passe dans les autres pays (CFPP2000, 12-03)

et également deux pour le sous-corpus des ESLO2, avec un pourcentage donc légèrement supérieur $(1,4 \%$ vs $2 \%)$.

Ces structures peuvent se retrouver dans les interrogatives directes du type (v. Lefeuvre \& Rossi-Gensane 2015) :

comment que ça s'appelle (CFPP2000, MO-02)

Nous analyserons ces structures comme venant de la même famille que les structures clivées :

(60) C'est comment que ça s'appelle?

(61) Comment est-ce que ça s'appelle?

Comme précédemment, il existe une tension entre, d'une part, le caractère proche d'une indépendante de la structure interrogative indirecte (celle-ci reproduisant la structure d'une interrogative directe) et, d'autre part, la confection de ce qui peut apparaître comme un subordonnant complexe, associant au mot en $q u$ - le que propre des clivées.

Ce schéma semble en régression dans le français hexagonal. C'est ce que permet de montrer une comparaison entre les ESLO1 et ESLO2, ces deux corpus 
ayant 40 années d'écart. Pour les ESLO1, nous avons trouvé 49 exemples de ce type (tout verbe introducteur) alors que, pour les ESLO2 (qui comprend plus d'heures d'enregistrement que les ESLO1 ; 460 vs 319), seuls 33 exemples ont été dénombrés :

(62) je sais plus où qu'ils travaillaient (ESLO2_ENT_1030_C)

(63) la petite dernière je sais plus comment qu'elle s'appelle (ESLO2_REPAS_1264_C)

\subsubsection{Les interrogatives indirectes partielles « in situ »}

Nous n'avons décompté que deux interrogatives indirectes in situ dans le CFPP2000, dont une seule avec le verbe savoir (0,7 \%) :

euh il $\mathrm{m}^{\prime}$ dit "ben écoute $\mathrm{t}^{\prime}$ as encore trois trois autres entretiens entretiens médical psychologique et un autre entretien je sais plus c'était quoi la fin et plus un test un test [mm] euh + un test un questionnaire" (CFPP2000, SO-02)

Dans le sous-corpus Les repas tiré des ESLO2, on en dénombre 5, avec le verbe savoir, ce qui donne un pourcentage nettement supérieur (5\% vs 0,7\%) :

c'est vachement bon le poulet comme ça je sais pas c'est quoi mais (ESLO2 REPAS 1259)

(66) moi je je me je me je me suis pas inscrit donc je sais pas c'est quand le jour (ESLO2_REPAS_1259)

(67) bah Yann tu sais pas c'est qui il est chauve un peu hein (ESLO2_REPAS_1257)

Nous n'avons pas trouvé de c'est quoi ou c'est qui après le verbe demander dans le corpus d'Orléans.

On peut supposer que le registre, plus informel dans le sous-corpus Les repas, ainsi que la jeunesse des interlocuteurs (le plus âgé a 29 ans) favorisent ce type de productions langagières. Dans le CFPP2000, le seul exemple répertorié est également prononcé par un locuteur jeune (29 ans). Le corpus des ESLO1 ne comporte aucun exemple d'interrogatives indirectes in situ.

Le schéma in situ semble directement calqué sur celui des interrogatives directes (v. Lefeuvre 2015) :

$$
\text { C'était quoi? }
$$

Pour B. Defrancq (2000 : 132), ce sont des « structures sans élément subordonnant », alors que « la structure à enchâssement se caractérise [...] par la présence d'un élément subordonnant ». Cependant, l'enchâssement ne se fait pas nécessairement à l'aide d'un subordonnant. On peut supposer, comme il a déjà été évoqué, que d'autres facteurs interviennent, tels que le contexte et la prosodie.

Les structures in situ se développent particulièrement avec le pronom quoi, sans doute en raison du fait que ce mot ne peut pas introduire une interrogative indirecte (sauf s'il est précédé d'une préposition) (v. Lefeuvre 2006) :

(69) *je sais plus quoi c'était. 
L'exemple suivant procure une variante du schéma in situ :

mais enfin vous me demandez si si si je faisais quoi alors pour cela? (ESLO1_ENT_052_C)

La structure est introduite par si, démarcatif ouvrant une interrogative indirecte (totale) qu'il ait, ou non, le statut de subordonnant; quoi correspondrait à un pronom indéfini objet. L'énoncé standard :

(71) vous me demandez ce que je faisais

ne renferme pas quoi, agrammatical dans cette configuration :

(72) *vous me demandez quoi je faisais.

Il convient de préciser que cette question " entretient une relation avec son contexte antérieur », même s'il ne s'agit pas du " contexte antérieur immédiat » (Kerbrat-Orecchioni, $1991: 21$ ), car elle « reprend » une autre question posée par l'enquêteur quelques minutes auparavant (quand vous écriviez des mots d'excuse à l'école pour vos enfants est-ce que vous faisiez un brouillon ?). Elle entre donc, selon A. Borillo (1978 : 674 sqq. ; citée par Kerbrat-Orecchioni, 1991 : 21), dans la catégorie des "questions-répliques ", plus particulièrement des " questionssuspens » (de la sorte Tu as vu Pierre hier ? - Si j'ai vu qui ?, exemple emprunté à Kerbrat-Orecchioni, $1991: 21$ ).

Un rapprochement peut être effectué avec certaines occurrences dans des français d'Afrique, comme l'exemple suivant tiré de la presse malienne (qui n'est pas une " question-réplique »), où si ouvre une interrogative indirecte apparemment totale, mais comportant néanmoins les pronoms qui et quoi de l'interrogation partielle « non extraits » :

(73) M. Traoré martèle ses mots quand il se pose la question de savoir s'ils représentent qui ou quoi (M-L'aurore, 01/06/1992 in Dagnac 1996 ; citée par Ngamountsika, 2013 : 168)

Nous ne pensons donc pas, contrairement à B. Defrancq (2000 : 137), que le développement de la structure in situ s'explique " par un problème de syntaxe locale qui touche la structure à attribut », mais que, plus généralement, cette structure in situ est à rapporter au problème que connaît le français pour les emplois du pronom quoi (v. Lefeuvre 2006), ainsi qu'à la construction des interrogatives indirectes qui s'établit en reproduisant le schéma des interrogatives directes.

\subsubsection{Autres schémas : avec inversion, "qui c'est qui »}

F. Gadet (1989 : 140) relève des énoncés comme :

(74) Je me demande quand part-il.

Ce type d'énoncés avec inversion semble typique de conduites d'hypercorrection. Le locuteur « en fait trop » : il essaie de produire une forme d'interrogation qui relève $d^{\prime}$ 'un usage soutenu en inversant le verbe et le sujet mais, en alignant 
l'interrogative sur sa forme la plus normée, il oublie qu'il n'y a pas d'inversion dans la subordonnée. On entend parfois ces formes à la radio ou à la télévision et on les trouve à l'écrit dans des copies d'étudiants. Dans la situation d'enregistrement du CFPP2000, les locuteurs n'ont pas jugé utile d'avoir recours au registre le plus soutenu. Ces structures n'y sont pas attestées. De même, B. Defrancq (2000 : 137-138) n'en a pas rencontré dans le corpus oral CorpAix.

N'apparaît pas non plus dans le CFPP2000 ni dans le sous-corpus des ESLO2_Les repas le schéma en qui c'est qui. Cette structure est présente sporadiquement dans les ESLO2 (5 résultats, tous avec qui) :

(75) bon l'accouchement elle sait qui c'est qui a accouché sa mère l'a dit (ESLO2_ENT_1083_C)

(76) oui oui oui oh je sais pas qui c'est qui avait fait courir ça (ESLO2_ENT_1006_C)

\section{CONCLUSION}

Du point de vue des données, on a pu voir que le schéma standard des interrogatives indirectes est de loin le plus représenté (autour de $90 \%$ ) dans les corpus examinés. La variation des interrogatives indirectes en registre informel existe donc à la limite du système.

La présence d'interrogatives indirectes totales, pour lesquelles si et des ligateurs énonciatifs sont contigus, pourrait amener à considérer si comme une balise de discours. De même, l'existence de quelques interrogatives indirectes totales sans $s i$, d'interrogatives indirectes partielles mais également totales en est-ce $q u e$, et $\mathrm{d}$ 'interrogatives indirectes partielles, plus récentes, in situ, toutes proches des schémas des interrogatives directes, autorise à penser que les interrogatives indirectes non standard sont relativement autonomes, sans (nécessairement de) subordonnant introducteur, et avec un lien d'enchâssement qui s'établirait en discours, le cas échéant par la prosodie. Dans une conception privilégiant un traitement uniforme des interrogatives indirectes, standard comme non standard, cette constatation va à l'appui de l'analyse selon laquelle, de manière générale, les interrogatives indirectes sont des subordonnées sans subordonnant.

Enfin, si, à la faveur de quelques exemples, un rapprochement, dans le domaine de l'interrogation indirecte, a été esquissé avec les français parlés en Afrique, la piste d'une proximité entre vernaculaires d'une même langue, notamment à la suite de B. Boutin et F. Gadet (2012), reste à approfondir.

\section{Références bibliographiques}

[CFPP2000] Discours sur la ville. Corpus de Français Parlé Parisien des années 2000. [http://cfpp2000.univ-paris3.fr/]

[ESLO] Enquêtes SocioLinguistiques à Orléans. [http://eslo.huma-num.fr/index.php] 
[FRANTEXT] Base textuelle Frantext, ATILF (CNRS \& Université de Lorraine). [http://www.frantext. $\mathrm{fr} /]$

ANDERSEN H. L. (2000), " Discours rapporté en français parlé : rection du verbe de citation et éléments définissant la citation directe ", Études romanes 47, 143-155.

ARRIVÉ M., GAdet F. \& Galmiche M. (1986), La grammaire d'aujourd'hui, Paris, Flammarion.

BLANCHE-BENVENISTE C. (1989), "Constructions verbales <en incise> et rection faible des verbes ", Recherches sur le français parlé 9, 53-73.

BOUtin B. A. \& GADET F. (2012), "Comment ce que montrent les français d'Afrique s'inscrit/ne s'inscrit pas dans les dynamiques des français dans une perspective de francophonie ", Le français en Afrique 27, 19-34.

Boutin B. A. \& Rossi-Gensane N. (2015), "Quelle(s) diversité(s) pour la syntaxe ? ", in M. Abecassis \& G. Ledegen (éds), De la genèse de la langue à Internet : variations dans les formes, les modalités et les langues en contact, Berne, Peter Lang, 155-178.

Branca S. \& Lefeuvre F. (2016), "Le Corpus de Français Parlé Parisien des années 2000 : constitution, outils et analyses. Le cas des interrogatives indirectes ", Corpus 15, 265-284.

Branca-Rosoff S. et al. (2012), Discours sur la ville. Corpus de Français Parlé Parisien des années 2000 (CFPP2000). [http://cfpp2000.univ-paris3.fr/CFPP2000.pdf]

COVEnEY A. (2011), "L'interrogation directe ", Travaux de linguistique 63 (2), 112-145.

DAGNAC A. (2014), "La variation des interrogatives en français ", Document préparatoire (texte provisoire) pour contribution à la Grande Grammaire du Français. [https://hal.archivesouvertes.fr/hal-00988751]

DEFRANCQ B. (2000), "Un aspect de la subordination en français parlé : l'interrogation indirecte ", Études romanes 47, 131-141.

DefrancQ B. (2005), L'interrogative enchâssée : structure et interprétation, Bruxelles, De Boeck/Duculot.

GACHET F. (2015), Incises de discours rapporté et autres verbes parenthétiques : étude grammaticale, Paris, Honoré Champion.

GADET F. (1989), Le français ordinaire, Paris, Armand Colin.

GADET F. (1997), "La variation, plus qu'une écume ", Langue française 115, 5-18.

GADET F. (2010), "Sociolinguiste dans une grammaire : la variation pour une grammaire du français ", in M. Iliescu, H. Siller-Runggaldier \& P. Danler (éds), XXVe CILPR - Congrès International de Linguistique et Philologie Romanes, 3-8 septembre 2007, Innsbruck, t. IV, Berlin/New York, De Gruyter, 117-125.

Hadermann P. (1993), Étude morphosyntaxique du mot " où ", Paris/Louvain-la-Neuve, Duculot.

KeRBRAT-ORECCHIONI C. (1991), "Introduction ", in C. Kerbrat-Orecchioni (éd.), La question, Lyon, Presses Universitaires de Lyon, 5-37.

LE GOFFIC P. (1987), "Sur l'ambiguïté des relatives / interrogatives indirectes en ce qui, ce que ", in C. Fuchs (éd.), L'ambiguïté et la paraphrase : opérations linguistiques, processus cognitifs, traitements automatisés, Caen, Centre de publication de I'Université de Caen, 83-88.

LE GofFIC P. (1993), Grammaire de la phrase française, Paris, Hachette.

Le QUERLER N. (1997), "La place du sujet nominal dans les subordonnées percontatives ", in C. Fuchs (éd.), La place du sujet en français contemporain, Louvain-la-Neuve, Duculot, 179-203. 
LEDEGEN G. (2007), "L'interrogative indirecte in situ à la Réunion : elle connaît elle veut quoi ", in M. Abecassis, L. Ayosso \& É. Vialleton (éds), Le français parlé $d u X X X^{e}$ siècle : normes et variations géographiques et sociales, Paris, L'Harmattan, 177-200.

LEFEUVRE F. (2006), Quoi de neuf sur " quoi " ? Étude morphosyntaxique du mot " quoi ", Rennes, Presses Universitaires de Rennes.

LEFEUVRE F. (2014), Étude grammaticale du français classique à partir de textes, Paris, Presses de la Sorbonne Nouvelle.

LEFEUVRE F. (2015), "Analyse grammaticale et sur corpus de l'expression c'est quoi (?) ", in G. Dostie \& P. Hadermann (éds), La dia-variation en français actuel : études sur corpus, approches croisées et ouvrages de référence, Berne, Peter Lang, 39-62.

LefeuvRe F. \& Rossi-Gensane N. (2015), " Interrogation ", in P. Larrivée \& F. Lefeuvre (dir.), Projet Fracov. [http://www.univ-paris3.fr/index-des-fiches-227311.kjsp?RH=1373703153287]

MARTIN R. (1985), "L'interrogation comme universel du langage ", in P. Valentin (éd.), L'interrogation, Paris, Presses de l'Université de Paris-Sorbonne, 257-284.

Morel M.-A. \& DANON-Bolleau L. (1998), Grammaire de l'intonation : l'exemple du français, Paris, Ophrys.

NGAMOUNTSIKA É. (2013), Le discours rapporté dans l'oral spontané : l'exemple du français parlé en République du Congo, Pessac, Presses Universitaires de Bordeaux.

Riegel M., Pellat J.-C. \& Rioul R. $\left(2009^{7}\right)$, Grammaire méthodique du français, Paris, Presses Universitaires de France.

RosieR L. (1999), Le discours rapporté : histoire, théories, pratiques, Bruxelles, Duculot.

ROSIER L. (2008), Le discours rapporté en français, Paris, Ophrys.

SCHneIDER S. (2007), Reduced Parenthetical Clauses as Mitigators: A Corpus Study of Spoken French, Italian and Spanish, Amsterdam, Benjamins.

SERBAT G. (1985), "Le verbe introducteur de la subordonnée interrogative en latin ", in P. Valentin (éd.), L'interrogation, Paris, Presses de l'Université de Paris-Sorbonne, 3-16.

VET C. (1994), "Savoir et croire ", Langue française 102, 56-68.

WILMET M. $\left(2003^{3}\right)$, Grammaire critique du français, Bruxelles, Duculot.

Wimmer C. (1983), "Syntaxe et interprétation de la structure <V si p > (interrogative indirecte) ", Le français moderne 51 (3), 205-223. 